Article

\title{
Human Dermal Stem/Progenitor Cell-Derived Conditioned Medium Improves Senescent Human Dermal Fibroblasts
}

\author{
Ji-Yong Jung ${ }^{\dagger}$, Joong Hyun Shim ${ }^{\dagger}$, Hyun Choi, Tae Ryong Lee * and Dong Wook Shin * \\ Amorepacific Corporation R\&D Center, 314-1 Bora-dong, Giheung-gu, Yongin-si, \\ Geyonggi-do 446729, Korea; E-Mails: ninefog@amorepacific.com (J.-Y.J.); \\ ungstrong@hanmail.net (J.H.S.); happycell@amorepacific.com (H.C.) \\ $\dagger$ These authors contributed equally to this work. \\ * Authors to whom correspondence should be addressed; \\ E-Mails: TRLee@amorepacific.com (T.R.L.); biopang@empal.com (D.W.S.); \\ Tel.: +82-31-280-5850 (T.R.L.); +82-10-2645-6496 (D.W.S.); \\ Fax: +82-31-899-2595 (T.R.L. \& D.W.S.).
}

Academic Editor: Wilhelm Bloch

Received: 6 July 2015/ Accepted: 27 July 2015/ Published: 13 August 2015

\begin{abstract}
Adult skin stem cells are recognized as potential therapeutics to rejuvenate aged skin. We previously demonstrated that human dermal stem/progenitor cells (hDSPCs) with multipotent capacity could be enriched from human dermal fibroblasts using collagen type IV. However, the effects of hDSPCs on cellular senescence remain to be elucidated. In the present study, we investigated whether conditioned medium (CM) collected from hDSPC cultures (hDSPC-CM) exhibits beneficial effects on senescent fibroblasts. We found that hDSPC-CM promoted proliferation and decreased the expression level of senescence-associated $\beta$-galactosidase in senescent fibroblasts. In addition, p53 phosphorylation and p21 expression were significantly reduced in senescent fibroblasts treated with hDSPC-CM. hDSPC-CM restored the expression levels of collagen type I, collagen type III, and tissue inhibitor of metalloproteinase, and antagonized the increase of matrix metalloproteinase 1 expression. Finally, we demonstrated that hDSPC-CM significantly reduced reactive oxygen species levels by specifically up-regulating the expression level of superoxide dismutase 2 . Taken together, these data suggest that hDSPC-CM can be applied as a potential therapeutic agent for improving human aged skin.
\end{abstract}


Keywords: fibroblast; human dermal stem/progenitor cells; senescence

\section{Introduction}

Normal somatic cells cultured in vitro display a limited capacity to divide, and thus eventually become senescent [1]. Various harmful signals, including oxidative stress, DNA damage, and telomere shortening, have been reported to promote cellular senescence [2,3]. This cellular status is characterized by growth arrest, enlarged cell size, increased expression of senescence-associated $\beta$-galactosidase (SA- $\beta$-Gal), and accumulation of the tumor suppressors p53 and p21, which participate in cell cycle arrest [4,5]. Cellular senescence is closely related to the aging process; the number of senescent cells gradually increases with age, and this accumulation contributes to tissue aging and the development of age-related diseases [6].

Adult stem cells are indispensable for maintaining homeostasis in all tissues of the body. Representative characteristics of these stem cells are their self-renewal and multipotent capacities [7-18]. These cells have been identified in various tissues, including the bone marrow [7-9], skeletal muscle [10], heart [11], adipose tissue [12], and skin [13-18]. We previously demonstrated that collagen type IV-enriched human dermal stem/progenitor cells (hDSPCs) exhibit stem cell-like characteristics [17]. We also recently reported that conditioned medium (CM) collected from hDSPCs (hDSPC-CM) improved human dermal fibroblasts (HDFs) damaged by ultraviolet A (UVA), which causes photoaging of the skin [19].

In the present study, we expanded on these findings and examined whether hDSPC-CM exerts beneficial effects on the cellular senescence that occurs in aging skin. We found that hDSPC-CM significantly increased proliferation, and reduced SA- $\beta$-gal expression, p53 phosphorylation, and reactive oxygen species (ROS) levels in senescent fibroblasts. These data suggest that hDSPC-CM is a potential stem cell-based therapeutic agent to prevent aging in the skin.

\section{Results}

\section{1. hDSPC-CM Increased Cell Proliferation in Senescent Fibroblasts}

We previously demonstrated that hDSPC-CM could restore UVA-induced damage in HDFs [19]. In the current study, we investigated whether hDSPC-CM would similarly reverse cellular senescence. First, we evaluated the effects of hDSPC-CM on the proliferation of senescent fibroblasts. We observed increased proliferation in senescent fibroblasts treated with hDSPC-CM compared with cells treated with non-hDSPC-CM (Figure 1A). A cell proliferation assay further revealed that hDSPC-CM promoted the proliferation of senescent fibroblasts in a concentration-dependent manner, whereas non-hDSPC-CM did not (Figure 1B). As expected, senescent fibroblasts expressed lower levels of the cell proliferation marker Ki67 compared with control fibroblasts (data not shown). However, treatment with hDSPC-CM, but not non-hDSPC-CM, significantly enhanced Ki67 expression in senescent fibroblasts (Figure 1C,D). 
A
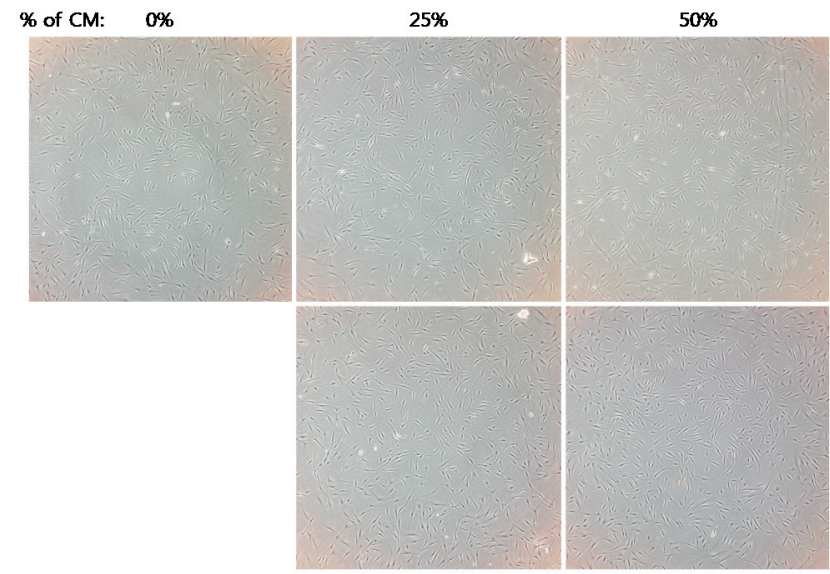

$100 \%$

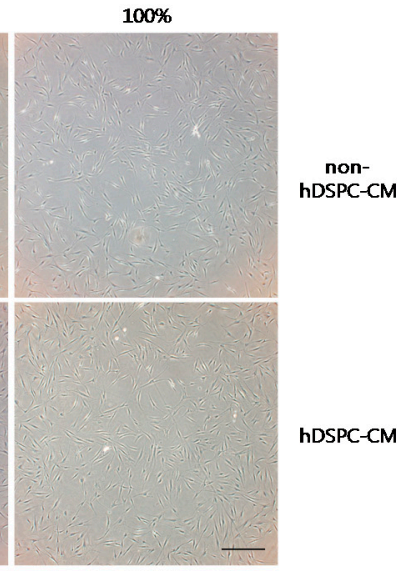

B

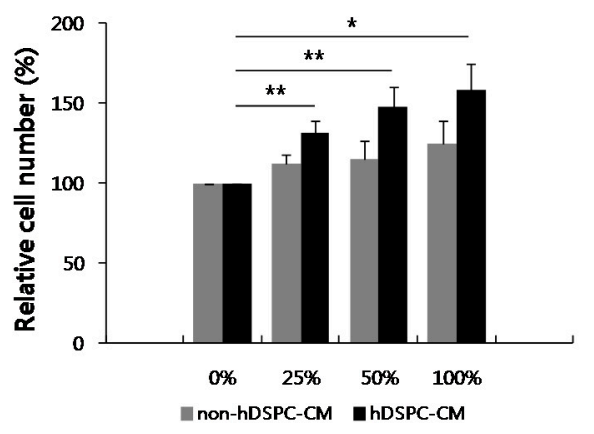

C

\section{D}
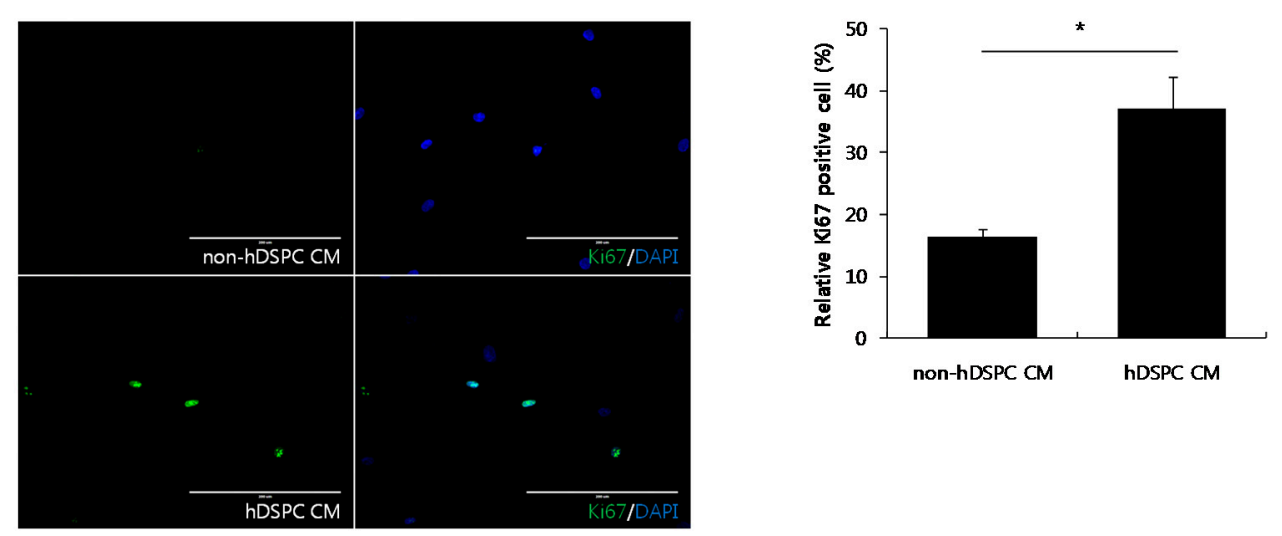

Figure 1. hDSPC-CM promoted proliferation in senescent fibroblasts. Senescent fibroblasts were treated with the indicated concentrations of either non-hDSPC-CM (upper panel) or hDSPC-CM (lower panel). Images were taken $24 \mathrm{~h}$ after CM treatment. The scale bar represents $500 \mu \mathrm{m}$ (A); Senescent fibroblasts were incubated with the indicated concentrations of each $\mathrm{CM}$ for $24 \mathrm{~h}$ and cell-counting assays were performed. Graphs depict the means \pm S.E.M of three independent experiments. ${ }^{*} p<0.05, * * p<0.01$ (B); Senescent fibroblasts were treated with 50\% of either non-hDSPC-CM or hDSPC-CM for $24 \mathrm{~h}$. Immunofluorescent staining assays were performed with anti-Ki67 (green) antibody and DAPI (blue). The scale bar represents $400 \mu \mathrm{m}$ (upper panel) or $200 \mu \mathrm{m}$ (lower panel) (C); Ki67-positive cells were quantified. The graph depicts means \pm S.E.M from three independent experiments. ${ }^{*} p<0.05$ (D). Data were statistically compared with the unpaired Student's $t$-test (B,D). 


\section{2. hDSPC-CM Inhibited Senescence-Related Signaling in Fibroblasts}

Next, we investigated whether hDSPC-CM could reverse cellular senescence. SA- $\beta$-Gal is a well-known marker of cellular senescence in various cells, including fibroblasts [20,21]. We observed higher SA- $\beta$-Gal expression in senescent fibroblasts compared to young fibroblasts (Figure 2A, upper panel). However, hDSPC-CM treatment significantly decreased the number of SA- $\beta$-Gal-positive cells relative to fresh medium $(0 \% \mathrm{CM})$ or non-hDSPC-CM treatment (Figure $2 \mathrm{~A}$, lower panel and $2 \mathrm{~B})$.

The tumor suppressor p53 regulates cell cycle arrest, apoptosis, and cellular senescence [2-5]. We thus investigated whether hDSPC-CM could inhibit the p53 signaling pathway. Treatment with $\mathrm{hDSPC}-\mathrm{CM}$ for $72 \mathrm{~h}$ reduced $\mathrm{p} 53$ phosphorylation. The expression level of $\mathrm{p} 21$, which is regulated by p53, was also inhibited by hDSPC-CM treatment (Figure 2C,D). In summary, our results suggest that hDSPC-CM could contribute to reduce cellular senescence by suppressing p53 signaling.

A

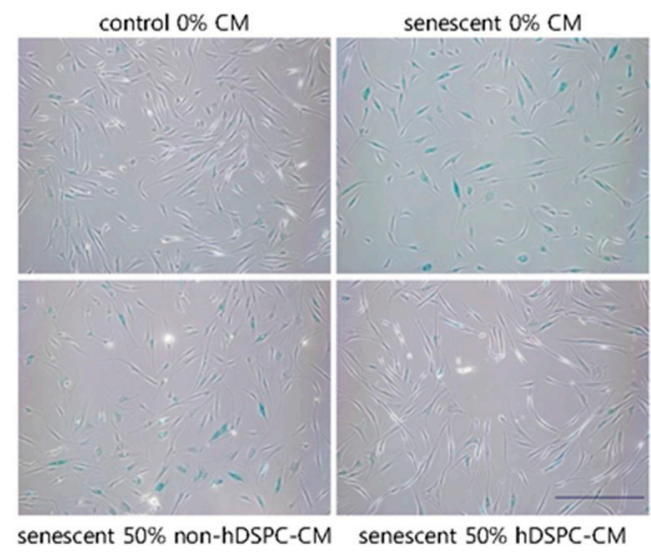

B

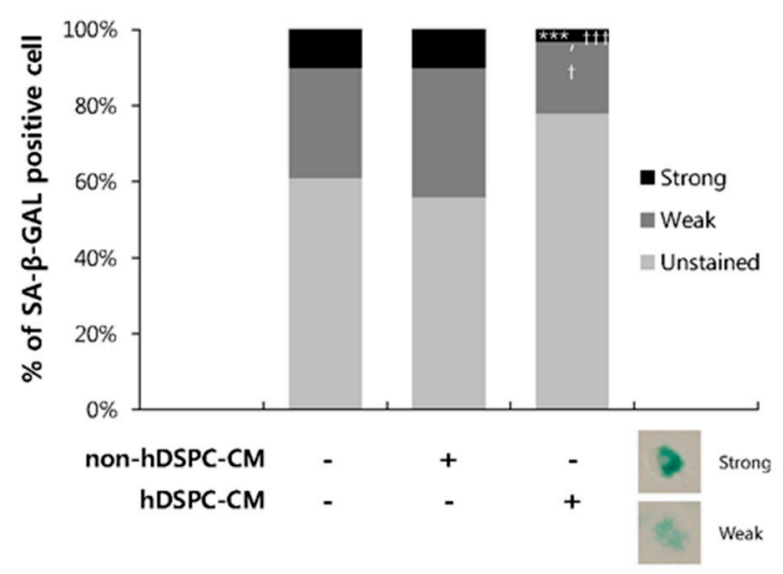

C

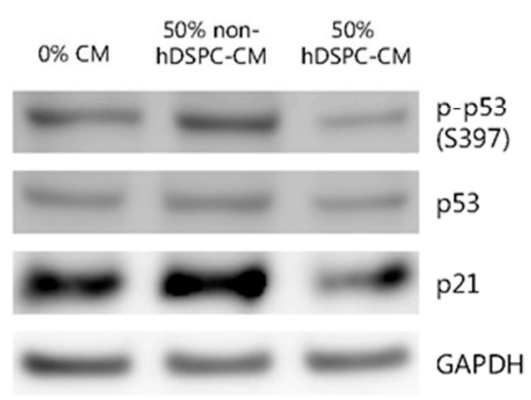

D

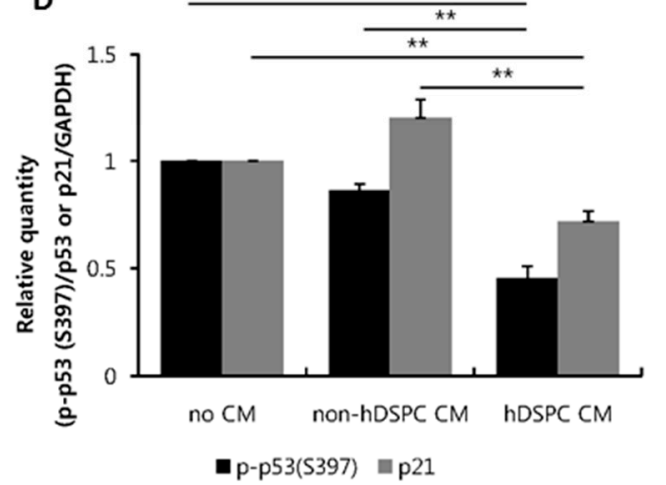

Figure 2. hDSPC-CM reduced the expression of senescence-associated markers in senescent fibroblasts. Senescent fibroblasts were incubated with either $0 \%$ or $50 \%$ of each CM for $72 \mathrm{~h}$. SA- $\beta$-Gal-positive cells appeared blue when visualized with optical microscopy. The scale bar indicates $500 \mu \mathrm{m}(\mathbf{A})$; Quantification of SA- $\beta$-Gal-positive cells in panel A. $* * * p<0.001$ vs. $0 \% \mathrm{CM}$ control; ${ }^{\dagger} p<0.05,{ }^{\dagger \dagger} p<0.001$ vs. non-hDSPC-CM group (B); Western blot analysis of p53 and p21 in each group of CM-treated senescent fibroblasts. The image represents three independent experiments and graphs depict means \pm S.E.M. ${ }^{* *} p<0.01$, $* * * p<0.001$ (C,D). Data were statistically compared with one-way analysis of variance (B) or an unpaired Student's $t$-test (D). 


\section{3. hDSPC-CM Enhanced HDF-Specific Markers}

We next investigated whether hDSPC-CM affects the expression levels of fibroblast-specific markers in senescent fibroblasts. The transcript levels of collagen type 1 alpha 1 (COL1A1), collagen type 3 alpha 1 (COL3A1), and tissue inhibitor of metalloproteinase (TIMP1), which are important components of the skin dermis, were significantly reduced in senescent fibroblasts compared with normal fibroblasts, and hDSPC-CM treatment significantly increased the expression levels of all three markers (Figure 3A,B and D). Conversely, matrix metalloproteinase 1 (MMP1) transcripts were increased in senescent fibroblasts compared with normal fibroblasts, and $\mathrm{hDSPC}-\mathrm{CM}$ treatment significantly reduced MMP1 expression (Figure 3C).

A

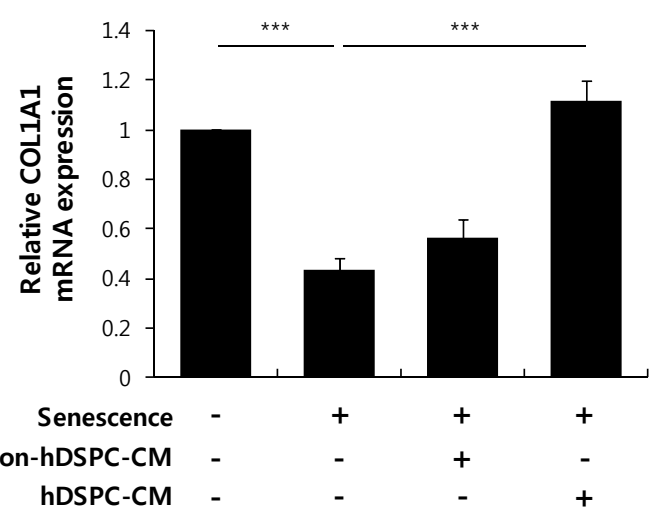

C

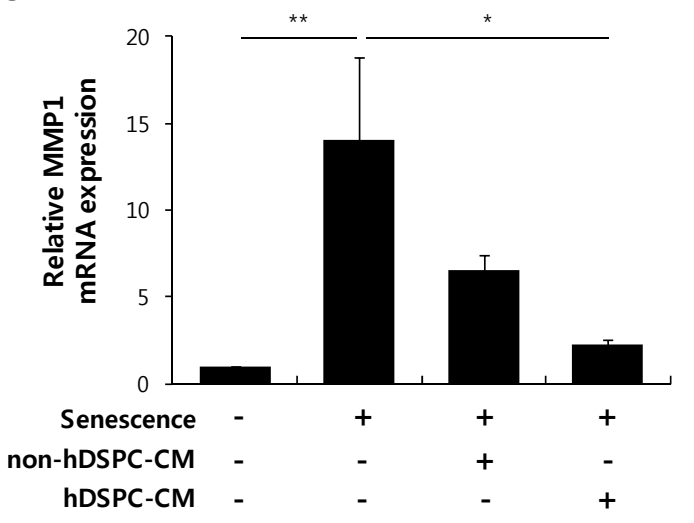

B

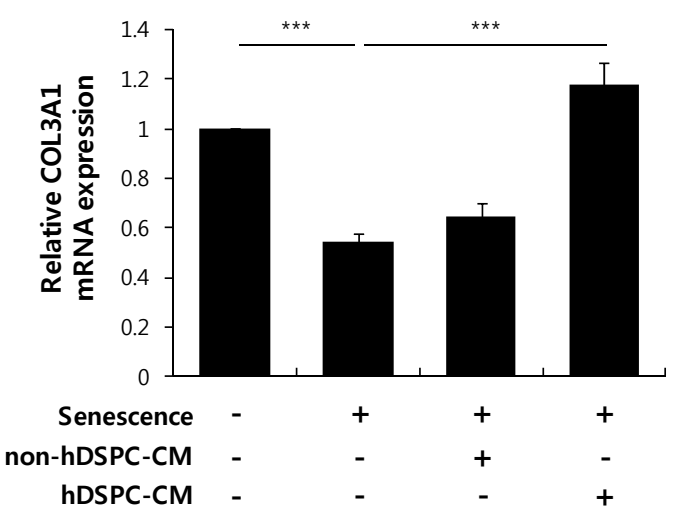

D

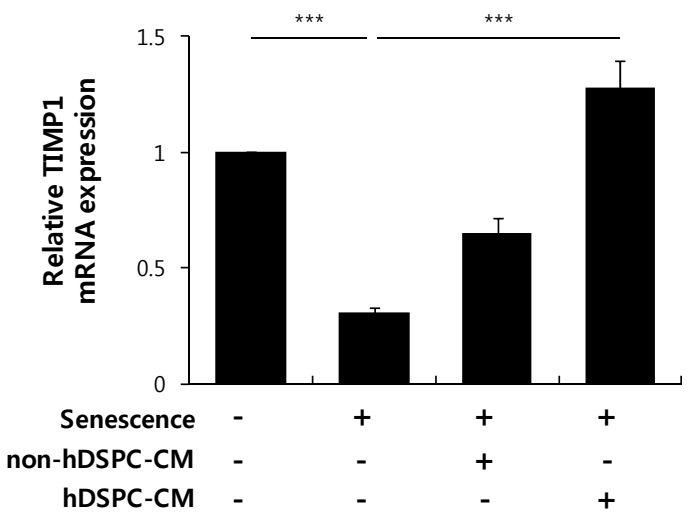

Figure 3. hDSPC-CM restored the expression of specific dermal makers in senescent fibroblasts. Senescent fibroblasts were treated with either hDSPC-CM or non-hDSPC-CM for $24 \mathrm{~h}$. Real-time RT-PCR analysis was performed to quantify the expression levels of COL1A1 (A); COL3A1 (B); MMP1 (C); and TIMP1 (D). The graphs depict means \pm S.E.M of eight independent experiments. ${ }^{*} p<0.05,{ }^{* *} p<0.01,{ }^{* * *} p<0.001$. Data were statistically compared with one-way analysis of variance.

\section{4. hDSPC-CM Suppressed $\mathrm{H}_{2} \mathrm{O}_{2}$ Generation in Senescent Fibroblasts}

Several studies have reported that increased oxidative stress causes cellular senescence and aging [22-24]. Therefore, we examined whether hDSPC-CM could suppress ROS generation in senescent fibroblasts. Senescent fibroblasts produced higher ROS levels than normal fibroblasts [22,23]. 
Although both non-hDSPC-CM and hDSPC-CM inhibited the level of $\mathrm{H}_{2} \mathrm{O}_{2}$ in senescent fibroblasts, hDSPC-CM treatment more significantly reduced $\mathrm{H}_{2} \mathrm{O}_{2}$ generation (Figure 4).

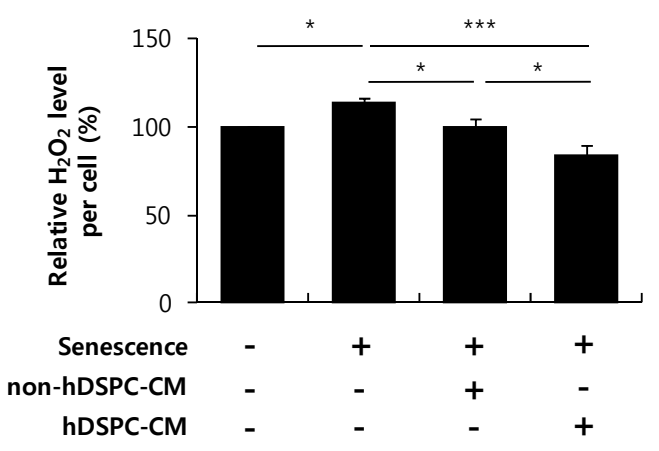

Figure 4. hDSPC-CM inhibited $\mathrm{H}_{2} \mathrm{O}_{2}$ generation in senescent fibroblasts. $\mathrm{Basal}_{2} \mathrm{H}_{2} \mathrm{O}_{2}$ levels in senescent fibroblasts were measured with Amplex Red fluorescent dye at $24 \mathrm{~h}$ after treatment with the indicated $\mathrm{CMs}$, as described in the Experimental Section. Graphs depict means \pm S.E.M of three independent experiments. $* p<0.05, * * * p<0.001$. Data were statistically compared with one-way analysis of variance.

\section{5. hDSPC-CM Specifically Increased the Superoxide Dismutase 2 (SOD2) Expression Level among} ROS Scavenging Enzymes in Senescent Fibroblasts

ROS scavenging enzymes are responsible for reducing $\mathrm{H}_{2} \mathrm{O}_{2}$ levels to maintain cell homeostasis [21-23]. Thus, we tested whether hDSPC-CM could increase the expression of ROS scavenging enzymes such as superoxide dismutases (SODs) and catalase. Interestingly, among the ROS scavenging enzymes tested, hDSPC-CM specifically enhanced the expression level of SOD2 in senescent fibroblasts (Figure 5).
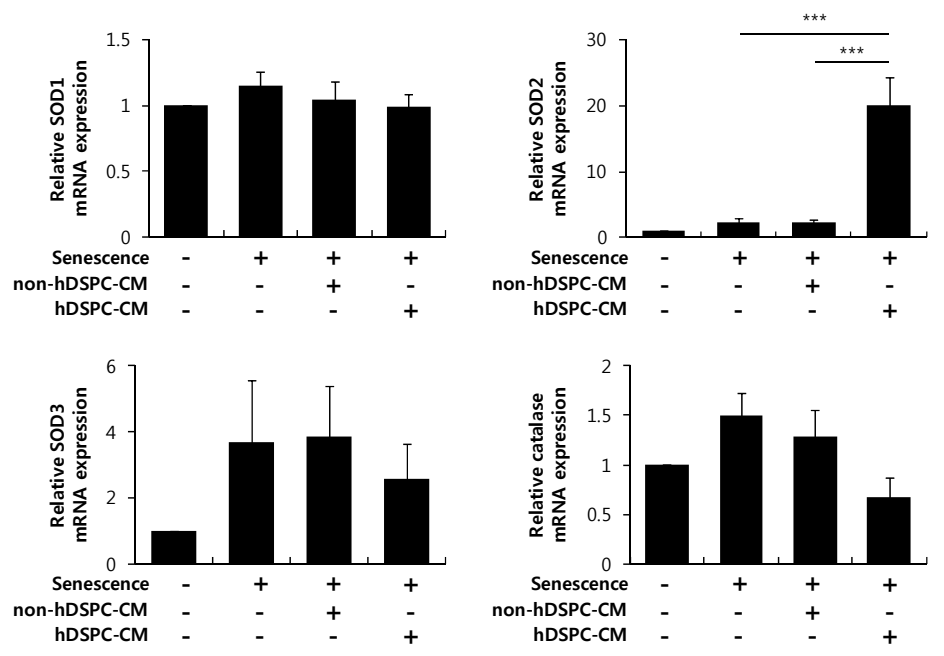

Figure 5. hDSPC-CM specifically up-regulated the expression level of the ROS scavenging enzyme SOD2 in senescent fibroblasts. Senescent fibroblasts were treated with either hDSPC-CM or non-hDSPC-CM for $24 \mathrm{~h}$. Real-time RT-PCR was performed for SOD1, $S O D 2, S O D 3$, and catalase. Graphs depict means \pm S.E.M of four independent experiments. $* * * p<0.001$. Data were compared with one-way analysis of variance. 


\section{Discussion}

The aging process gradually decreases the homeostatic and regenerative potential of all tissues [25-28]. In particular, photoaging and intrinsic aging decreases the elasticity of the skin, resulting in the formation of wrinkles and impairing wound healing [29-31]. These age-related changes might reflect the gradual increase in the proportion of senescent cells in specific tissues. Adult stem cells have been considered as potential therapeutics to slow this aging process. Indeed, we recently demonstrated that hDSPC-CM restored UVA-induced damages to fibroblasts [19].

In the present study, we further demonstrated that hDSPC-CM reversed multiple phenotypes associated with cellular senescence. First, we found that hDSPC-CM significantly enhanced the proliferation of senescent fibroblasts (Figure 1). Second, we found that hDSPC-CM significantly decreased SA- $\beta$-gal production, p53 phosphorylation, and the expression level of p21, all of which were generally increased in senescent fibroblasts (Figure 2). Third, RT-PCR analyses revealed that hDSPC-CM restored the expression of major dermal biomarkers, including COL1A1, COL3A1, and TIMP 1, which were typically down-regulated in senescent fibroblasts (Figure 3). Fourth, we demonstrated that hDSPC-CM significantly decreased $\mathrm{H}_{2} \mathrm{O}_{2}$ levels by specifically increasing SOD2 expression (Figures 4 and 5).

Mesenchymal stem cell (MSC) transplantation accelerates the wound-healing process in damaged skin [32-34]. Moreover, some MSC transplantation studies have suggested that the therapeutic potential of MSCs might be mediated by secreted growth factors rather than their long-term presence in injured tissues $[35,36]$. Recent studies demonstrated that CM harvested from stem cell cultures exerts beneficial effects on multiple cellular defects and diseases [37-39]. For example, Chen et al. demonstrated that cytokines present in murine bone marrow-derived MSC-CM stimulated macrophages, endothelial migration, and wound healing in BALB/c mice [37]. Similarly, another study showed that the paracrine effects of MSCs accelerated the regeneration of endogenous stem cells and ameliorated obstruction-induced overactive bladders [38]. Kim et al. reported that soluble intracellular adhesion molecule-1 secreted by human umbilical cord blood-derived MSCs reduced the number of amyloid- $\beta$ plaques, which can cause Alzheimer's disease [39]. In our previous study, cytokine array analysis revealed that hDSPC-CM contains higher levels of multiple growth factors, including basic fibroblast growth factor, hepatocyte growth factor, insulin-like growth factor-binding protein-1 and -2, insulin-like growth factor, and vascular endothelial growth factor, compared with non-hDSPC-CM [19]. Thus, we speculate that the beneficial effects of hDSPC-CM on senescent fibroblasts may be mediated by hDSPC-secreted growth factors.

According to previous reports, cell cycle regulators and DNA damage response pathways are strongly implicated in the onset of senescence [1-3,5,6,20]. For example, increased p53 phosphorylation forces cells into a state of premature senescence [2-4]; as cells enter G0, p21, a well-known target gene of p53, increases in a p53-dependent manner. Thus, we investigated the expression of these proteins and demonstrated that hDSPC-CM significantly down-regulated p53 phosphorylation as well as p21 expression (Figure 2C).

ROS are also considered to be major factors contributing to the aging process. Metabolic dysfunction and exogenous stress can generate excessive ROS levels [22-24]. Our data demonstrated that hDSPC-CM decreased $\mathrm{H}_{2} \mathrm{O}_{2}$ levels in senescent fibroblasts (Figure 4). To investigate the underlying mechanism to explain this phenomenon, we further examined the expression of several antioxidant 
enzymes, including members of the SOD family and catalase. According to previous reports, SOD1 is located in the cytoplasm, SOD2 is sequestered to the mitochondria, and SOD3 is secreted into the extracellular matrix [40,41]. Interestingly, we observed that only SOD2 expression was specifically enhanced in senescent fibroblasts cultured with hDSPC-CM compared with control and non-hDSPC-CM (Figure 5), implying that increased ROS production by senescent fibroblasts is mainly generated in the mitochondria $[40,41]$.

In conclusion, we suggest that hDSPC-CM ameliorates fibroblast senescence in a paracrine manner (Figure 6). Thus, the factors secreted by hDSPCs represent potential therapeutics to promote skin regeneration.

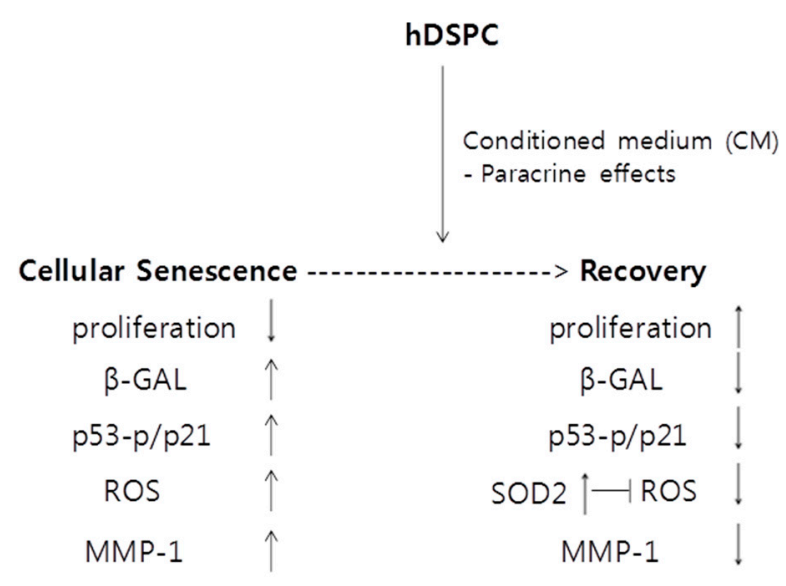

Figure 6. A schematic representation of the mechanism contributing to the beneficial effects of hDSPC-CM on senescent human dermal fibroblasts. $\uparrow$ means increase, whereas $\downarrow$ means decrease.

\section{Experimental Section}

\subsection{Preparation of $h D S P C-C M$}

HDFs (Lonza, Basel, Switzerland) were cultured in Dulbecco's modified eagle medium (DMEM) (Lonza) containing 10\% fetal bovine serum (FBS), $100 \mathrm{U} / \mathrm{mL}$ penicillin, and $100 \mu \mathrm{g} / \mathrm{mL}$ streptomycin (Life Sciences, St. Petersburg, FL, USA) at $5 \% \mathrm{CO}_{2}$ and $37{ }^{\circ} \mathrm{C}$. Fibroblasts were used within three passages.

To enrich hDSPCs that are present in only small amounts in fibroblasts, collagen type IV (Sigma-Aldrich, St. Louis, MO, USA)-coated dishes were prepared by coating 150-mm tissue culture dishes with collagen type IV $(20 \mathrm{~mL}, 20 \mu \mathrm{g} / \mathrm{mL})$ overnight at $4{ }^{\circ} \mathrm{C}$. We observed that $>50 \%$ of the HDFs adhered to the collagen type IV-coated dishes after $10 \mathrm{~min}$ of incubation. Therefore, we gradually reduced the adherence time in 1-min increments. Cells were not to adhere to dishes in less than 4 min. Ultimately, an incubation time of $12 \mathrm{~h}$ was selected because most of the cells could adhere to the dishes during this period. Thus, the cells were separated based on their ability to adhere to the plates within 4-5 min (hDSPCs) or within $12 \mathrm{~h}$ (non-hDSPCs) at $37{ }^{\circ} \mathrm{C}$. About $5 \%-10 \%$ of the total fibroblasts adhered to the dishes within $4-5$ min of incubation $[17,18]$. 
To obtain the CM, hDSPCs $\left(1 \times 10^{5}\right.$ cells $\left./ \mathrm{mL}\right)$ were cultured in a Hydrocell ${ }^{\mathrm{TM}}$ dish (Nunc, Penfield, NY, USA) with serum-free DMEM. After $48 \mathrm{~h}$ in culture, hDSPC-CM was collected, centrifuged at $300 \times \mathrm{g}$ for $5 \mathrm{~min}$, and filtered with a $0.22-\mu \mathrm{m}$ syringe filter (PALL) [19].

\subsection{Cell Culture and CM Treatment}

HDFs derived from adult skin (Cat. no. CC-2511) were purchased from Lonza and cultured in DMEM supplemented with 10\% FBS (Lonza), $100 \mathrm{U} / \mathrm{mL}$ penicillin, and $100 \mu \mathrm{g} / \mathrm{mL}$ streptomycin (Life Sciences) at $5 \% \mathrm{CO}_{2}$ and $37{ }^{\circ} \mathrm{C}$. Senescent fibroblasts were established as previously described [20,21].

For CM treatment, $3 \times 10^{5}$ cells were seeded onto $60-\mathrm{mm}$ dishes in the CM, and DMEM with $50 \%$ non-hDSPC-CM or 50\% hDSPC-CM was added to the cultures for the indicated times. All CMs were changed every $24 \mathrm{~h}$.

\subsection{Immunofluorescence Staining for Ki67}

Senescent fibroblasts were incubated with 50\% non-hDSPC-CM or hDSPC-CM for $24 \mathrm{~h}$. Following CM treatment, the cells were fixed with $4 \%$ formaldehyde (Sigma-Aldrich) and then blocked with 1\% bovine serum albumin (Sigma-Aldrich). The cells were then incubated with mouse monoclonal anti-Ki67 antibody (Abcam, Cambridge, MA, USA) for $12 \mathrm{~h}$ at $4{ }^{\circ} \mathrm{C}$, followed by Alexa-488-conjugated secondary antibody (Invitrogen, Carlsbad, CA, USA) for $1.5 \mathrm{~h}$ at room temperature. The nuclei were stained with $5 \mathrm{mg} / \mathrm{mL}$ DAPI (Sigma) for $5 \mathrm{~min}$. Samples were washed with phosphate-buffered saline with Tween-20 after each step. Slide-mounted samples were imaged with an EVOS fluorescence microscope (Advanced Microscopy Group, Mill Creek, WA, USA). Three images per dish were collected, and Ki67-positive cells were counted.

\subsection{Cell Proliferation Assay}

Senescent fibroblasts were seeded onto 96-well culture plates and treated with non-hDSPC-CM or $\mathrm{hDSPC}-\mathrm{CM}$ at the indicated dilutions for $24 \mathrm{~h}$. Following each CM treatment, $10 \mu \mathrm{L}$ of Cell Counting Kit- 8 (Sigma-Aldrich) was added to each well. After incubating for $1 \mathrm{~h}$, absorbance at $450 \mathrm{~nm}$ was measured.

\subsection{SA- $\beta-$ Gal Assay}

SA- $\beta$-Gal activity was detected using a senescence detection kit (Abcam) following the manufacturer's protocol. Briefly, senescent fibroblasts were treated with 50\% non-hDSPC-CM or $\mathrm{hDSPC}-\mathrm{CM}$ for $72 \mathrm{~h}$. The cells were then fixed and incubated at $37^{\circ} \mathrm{C}$ with a staining solution containing $1 \mathrm{mg} / \mathrm{mL}$ of 5-bromo-3-chloro-4-indolyl $\beta$-D-galactoside (X-gal) for $12 \mathrm{~h}$. Five images per dish were collected, and the blue cells were counted.

\subsection{Western Blot Analysis}

Senescent fibroblasts were incubated with 50\% non-hDSPC-CM or hDSPC-CM for $72 \mathrm{~h}$. The cells were lysed in RIPA buffer (Millipore, Billerica, MA, USA) supplemented with phosphatase inhibitors (Sigma-Aldrich) and proteinase inhibitors (Roche, Indianapolis, IN, USA). Protein lysates (20 $\mu$ g per 
lane) were run on $4 \%-12 \%$ Bis-Tris gels (Life Technologies, Carlsbad, CA, USA), transferred to polyvinylidene fluoride membranes (Roche), and analyzed by Western blotting. $\beta$-actin levels were measured in the samples as a control for protein loading using rabbit polyclonal anti- $\beta$-actin antibody (Santa Cruz Biotechnology, Santa Cruz, CA, USA). Phosphorylated and total p53 and p21 (Waf1/Cip1) levels were detected by rabbit polyclonal anti-phospho-p53 (serine 397), mouse monoclonal anti-p53, and rabbit monoclonal anti-p21, respectively (Cell Signaling Technology, Boston, MA, USA).

\subsection{RNA Isolation and Real-Time RT-PCR}

Total RNA was extracted using TRI Reagent ${ }^{\circledR}$ (Invitrogen) and quantified on a NanoDrop spectrophotometer (Thermo Scientific, Lafayette, CO, USA). Reverse transcription of $4 \mu \mathrm{g}$ total RNA was performed using ReverTra Ace reverse transcriptase (Toyobo, Osaka, Japan), and the reaction was terminated by adding Tris-EDTA buffer ( $\mathrm{pH} 8.0$ ) to $200 \mu \mathrm{L}$ of the cDNA (2 $\mu \mathrm{g})$ solution. Real-time RT-PCR was performed using a 7500 Fast Real-Time PCR System (Applied Biosystems, Foster City, CA, USA), according to the manufacturer's instructions. Briefly, $20 \mu \mathrm{L}$ of PCR mixture contained $10 \mu \mathrm{L}$ $2 \times \operatorname{TaqMan}^{\circledR}$ universal PCR Master Mix, $50 \mathrm{ng}$ cDNA, and $1 \mu \mathrm{L} 20 \times \operatorname{TaqMan}^{\circledR}$ Gene Expression assay reagent (Applied Biosystems). cDNA samples were analyzed to determine the expression levels of COL1A1, Hs00164004_m1; COL3A1, Hs00943809_m1; MMP1, Hs00899658_m1; TIMP1, Hs00171558_m1; SOD1, Hs00533490_m1; SOD2, Hs00167309_m1; and SOD3, Hs00156308_m1. Human GAPDH (43333764F) (Applied Biosystems) was used for normalizing the variation in cDNA quantities from different samples.

\section{8. $\mathrm{H}_{2} \mathrm{O}_{2}$ Production Measurements}

$\mathrm{H}_{2} \mathrm{O}_{2}$ released from senescent $\mathrm{HDFs}$ was measured using the Amplex Red Hydrogen Peroxide/Peroxidase Assay Kit (Life Technologies), according to the manufacturer's instructions. Senescent HDFs were treated with non hDSPC-CM or hDSPC-CM. The CM (50 $\mu \mathrm{L})$ of each sample was harvested and incubated with $100 \mu \mathrm{L}$ reaction solution containing $100 \mu \mathrm{M}$ Amplex Red reagent and $0.2 \mathrm{U} / \mathrm{mL}$ horseradish peroxidase for $10 \mathrm{~min}$. The fluorescence of each sample was measured at $590 \mathrm{~nm}$ emission following excitation at $560 \mathrm{~nm}$ using a Gemini XPS microplate reader (Molecular Device, Sunnyvale, CA, USA).

\subsection{Statistical Analysis}

Statistical analysis was performed using SPSS software (IBM Corporation, Armonk, NY, USA). An unpaired Student's $t$-test or one-way analysis of variance was used as indicated in the figure legends. $p$-values less than 0.05 were considered statistically significant.

\section{Conclusions}

For the first time, we demonstrated that CM derived from hDSPCs exhibits various anti-aging effects on senescent HDFs. hDSPC-CM not only reduced SA- $\beta$-gal levels but also decreased p53 phosphorylation and p21 expression in senescent HDFs. In addition, hDSPC-CM restored the expression of a major 
structural protein, collagen type $\mathrm{I}$, and reduced $\mathrm{H}_{2} \mathrm{O}_{2}$ levels in senescent $\mathrm{HDF}$. In conclusion, we suggest that hDSPC-CM can be clinically used as a potential therapeutic agent for improving human aged skin.

\section{Acknowledgments}

This study was partly supported by a grant from the Korea Healthcare Technology R \& D Project, Ministry of Health \& Welfare, Republic of Korea (Grant No.: A103017).

\section{Author Contributions}

Conceived and designed the experiments: Ji-Yong Jung, Joong Hyun Shim, and Dong Wook Shin. Performed the experiments: Ji-Yong Jung, Joong Hyun Shim, and Hyun Choi. Analyzed the data: Ji-Yong Jung, Joong Hyun Shim, and Hyun Choi. Wrote the paper: Tae Ryong Lee and Dong Wook Shin.

\section{Conflicts of Interest}

The authors declare no conflict of interest.

\section{References}

1. Pazolli, E.; Stewart, S.A. Senescence: The good the bad and the dysfunctional. Curr. Opin. Genet. Dev. 2008, 18, 42-47.

2. Roninson, I.B. Oncogenic functions of tumour suppressor $\mathrm{p} 21^{\mathrm{Waf1} / \mathrm{Cip} 1 / \mathrm{Sdi1}}$ : Association with cell senesence and tumour-promoting activities of stromal fibroblasts. Cancer Lett. 2002. 179, 1-14.

3. Collado, M.; Serrano, M. Senescence in tumours: Evidence from mice and humans. Nat. Rev. Cancer. 2010, 10, 51-57.

4. Toussaint, O.; Medrano, E.E.; von Zglinicki, T. Cellular and molecular mechanisms of stress-induced premature senescence (SIPS) of human diploid fibroblasts and melanocytes. Exp. Gerontol. 2000. 35, 927-945.

5. Alsayegh, K.N.; Gadepalli, V.S.; Iyer, S.; Rao, R.R. Knockdown of CDK2AP1 in primary human fibroblasts induces p53 dependent senescence. PLOS ONE 2015, 10, e0120782.

6. Campisi, J.; d'Adda di Fagagna, F. Cellular senescence: When bad things happen to good cells. Nat. Rev. Mol. Cell Biol. 2007, 8, 729-740.

7. Pereira, R.F.; Halford, K.W.; O’Hara, M.D .; Leeper, D.B.; Sokolov, B.P.; Pollard, M.D.; Bagasra, O.; Prockop, D.J. Cultured adherent cells from marrow can serve as long lasting precursor cells for bone, cartilage, and lung in irradiated mice. Proc. Natl. Acad. Sci. USA 1995, 92, 4857-4861.

8. Pittenger, M.F.; Mackay, A.M.; Beck, S.C.; Jaiswal, R.K.; Douglas, R.; Mosca, J.D.; Moorman, M.A.; Simonetti, D.W.; Craig, S.; Marshak, D.R. Multilineage potential of human mesenchymal stem cells. Science 1997, 284, 143-147.

9. Jiang, Y.; Jahagirdar, B.N.; Reinhardt, R.L.; Schwartz, R.E.; Keene, C.D.; Ortiz-Gonzalez, X.R.; Reyes, M.; Lenvik, T.; Lund, T.; Blackstad, M.; et al. Pluripotency of mesenchymal stem cells derived from adult marrow. Nature 2002, 418, 41-49. 
10. Jiang, Y.; Vaessen, B.; Lenvik, T.; Blackstad, M.; Reyes, M.; Verfaillie, C.M. Multipotent progenitor cells can be isolated from postnatal murine bone marrow, muscle, and brain. Exp. Hematol. 2002, 30, 896-904.

11. Yoon, J.; Choi, S.C.; Park, C.Y.; Shim, W.J.; Lim, D.S. Cardiac side population cells exhibit endothelial differentiation potential. Exp. Mol. Med. 2007, 39, 653-662.

12. Zuk, P.A.; Zhu, M.; Ashjian, P.; De Ugarte, D.A.; Huang, J.I.; Mizuno, H.; Alfonso, Z.C.; Fraser, J.K.; Benhaim, P.; Hedrick, M.H. Human adipose tissue is a source of multipotent stem cells. Mol. Biol. Cell 2002, 13, 4279-4295.

13. Blanpain, C.; Fuchs, E. Epidermal stem cells of the skin. Annu. Rev. Cell Dev. Biol. 2006, 22, 339-373.

14. Fuchs, E. Finding one's niche in the skin. Cell Stem Cell 2009, 4, 499-502.

15. Toma, J.G.; Akhavan, M.; Fernandes, K.J.; Barnabé-Heider, F.; Sadikot, A.; Kaplan, D.R.; Miller, F.D. Isolation of multipotent adult stem cells from the dermis of mammalian skin. Nat. Cell Biol. 2001, $3,778-784$.

16. Toma, J.G.; McKenzie, I.A.; Bagli, D.; Miller, F.D. Isolation and characterization of multipotent skin-derived precursors from human skin. Stem Cells 2005, 23, 727-737.

17. Shim, J.H.; Kang, H.H.; Lee, T.R.; Shin, D.W. Enrichment and characterization of human dermal stem/progenitor cells using collagen type IV. J. Dermatol. Sci. 2012, 67, 202-205.

18. Shim, J.H.; Lee, T.R.; Shin, D.W. Enrichment and characterization of human dermal stem/progenitor cells by intracellular granularity. Stem Cells Dev. 2013, 22, 1264-1274.

19. Shim, J.H.; Park, J.Y.; Lee, M.G.; Kang, H.H.; Lee, T.R.; Shin, D.W. Human dermal stem/progenitor cell-derived conditioned medium ameliorates ultraviolet a-induced damage of normal human dermal fibroblasts. PLoS ONE 2013, 8, e67604.

20. Kim, Y.M.; Byun, H.O.; Jee, B.A.; Cho, H.; Seo, Y.H.; Kim, Y.S.; Park, M.H.; Chung, H.Y.; Woo, H.G.; Yoon, G. Implications of time-series gene expression profiles of replicative senescence. Aging Cell 2013, 12, 622-634.

21. Dimri, G.P.; Lee, X.; Basile, G.; Acosta, M.; Scott, G.; Roskelley, C.; Medrano, E.E.; Linskens, M.; Rubelj, I.; Pereira-Smith, O. A biomarker that identifies senescent human cells in culture and in aging skin in vivo. Proc. Natl. Acad. Sci. USA 1995, 92, 9363-9367.

22. Finkel, T.; Holbrook, N.J. Oxidants, oxidative stress and the biology of aging. Nature 2009, 9, 239-247.

23. Liochev, S.I. Reactive oxygen species and the free radical theory of aging. Free Radic. Biol. Med. 2013, 60, 1-4.

24. Wondrak, G.T.; Roberts, M.J.; Cervantes-Laurean, D.; Jacobson, M.K.; Jacobson, E.L. Proteins of the extracellular matrix are sensitizers of photo-oxidative stress in human skin cells. J. Investig. Dermatol. 2003, 121, 578-586.

25. Kirkwood, T.B. Understanding the odd science of aging. Cell 2005, 120, 437-447.

26. Kirkwood, T.B. Time of our lives. What controls the length of life? EMBO Rep. 2005, 6, S4-S8.

27. Dorshkind, K.; Montecino-Rodriguez, E.; Signer, R.A. The ageing immune system: Is it ever too old to become young again? Nat. Rev. Immunol. 2009, 9, 57-62.

28. Dorshkind, K.; Swain, S. Age-associated declines in immune system development and function: Causes, consequences, and reversal Curr. Opin. Immunol. 2009, 21, 404-407. 
29. Gilchrest, B.A. A review of skin ageing and its medical therapy. Br. J. Dermatol. 1996, 135, 867-875.

30. Scharffetter-Kochanek, K.; Brenneisen, P.; Wenk, J.; Herrmann, G.; Ma, W.; Kuhr, L.; Meewes, C.; Wlaschek, M. Photoaging of the skin from phenotype to mechanisms. Exp. Gerontol. 2005, 35, 307-316.

31. Yaar, M.; Gilchrest, B.A. Photoageing: Mechanism, prevention and therapy. Br. J. Dermatol. 2007, $157,874-887$.

32. Sasaki, M.; Abe, R.; Fujita, Y.; Ando, S.; Inokuma, D.; Shimizu, H. Mesenchymal stem cells are recruited into wounded skin and contribute to wound repair by transdifferentiation into multiple skin cell type. J. Immunol. 2008, 180, 2581-2587.

33. Falanga, V.; Iwamoto, S.; Chartier, M.; Yufit, T.; Butmarc, J.; Kouttab, N.; Shrayer, D.; Carson, P. Autologous bone marrow-derived cultured mesenchymal stem cells delivered in a fibrin spray accelerate healing in murine and human cutaneous wounds. Tissue Eng. 2007, 13, 1299-1312.

34. Vojtassák, J.; Danisovic, L.; Kubes, M.; Bakos, D.; Jarábek, L.; Ulicná, M.; Blasko, M. Autologous biograft and mesenchymal stem cells in treatment of the diabetic foot. Neuro Endocrinol. Lett. 2006, 2, 134-137.

35. Van Koppen, A.; Joles, J.A.; van Balkom, B.W.; Lim, S.K.; de Kleijn, D.; Giles, R.H.; Verhaar, M.C. Human embryonic mesenchymal stem cell-derived conditioned medium rescues kidney function in rats with established chronic kidney disease. PLOS ONE 2012, 7, e38746.

36. Iso, Y.; Spees, J.L.; Serrano, C.; Bakondi, B.; Pochampally, R.; Song, Y.H.; Sobel, B.E.; Delafontaine, P.; Prockop, D.J. Multipotent human stromal cells improve cardiac function after myocardial infarction in mice without long-term engraftment. Biochem. Biophys. Res. Commun. 2007, 354, 700-706.

37. Chen, L.; Tredget, E.E.; Wu, P.Y.; Wu, Y. Paracrine factors of mesenchymal stem cells recruit macrophages and endothelial lineage cells and enhance wound healing. PLoS ONE 2008, 3, e1886.

38. Song, M.; Heo, J.; Chun, J.Y.; Bae, H.S.; Kang, J.W.; Kang, H.; Cho, Y.M.; Kim, S.W.; Shin, D.M.; Choo, M.S. The paracrine effects of mesenchymal stem cells stimulate the regeneration capacity of endogenous stem cells in the repair of a bladder-outlet-obstruction-induced overactive bladder. Stem Cells Dev. 2014, 23, 654-663.

39. Kim, J.Y.; Kim, D.H.; Kim, J.H.; Lee, D.; Jeon, H.B.; Kwon, S.J.; Kim, S.M.; Yoo, Y.J.; Lee, E.H.; Choi, S.J.; et al. Soluble intracellular adhesion molecule-1 secreted by human umbilical cord blood-derived mesenchymal stem cell reduces amyloid- $\beta$ plaques. Cell Death Differ. 2012, 19, 680-691.

40. Zelko, I.N.; Mariani, T.J.; Folz, R.J. Superoxide dismutase multigene family: A comparison of the CuZn-SOD (SOD1), Mn-SOD (SOD2), and EC-SOD (SOD3) gene structures, evolution, and expression. Free Radic. Biol. Med. 2002, 33, 337-349.

41. Fukai, T.; Ushio-Fukai, M. Superoxide dismutases: Role in redox signaling, vascular function, and diseases. Antioxid. Redox Signal. 2011, 15, 1583-1606.

(C) 2015 by the authors; licensee MDPI, Basel, Switzerland. This article is an open access article distributed under the terms and conditions of the Creative Commons Attribution license (http://creativecommons.org/licenses/by/4.0/). 